\title{
REVIEW
}

\section{An Integrative Review of Curricular Considerations for Preparing Student Pharmacists to Prescribe Hormonal Contraception}

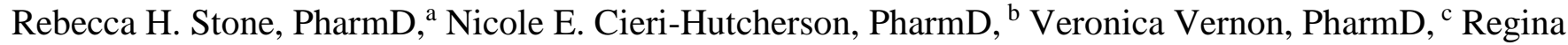 \\ Arellano, PharmD, ${ }^{\mathrm{d}}$ Cortney Mospan, ${ }^{\mathrm{e}}$ John Brock Harris, PharmD, ${ }^{\mathrm{e}}$ Kylie N. Barnes, PharmD, ${ }^{\mathrm{f}}$ Brooke L. \\ PharmD $^{\mathrm{d}}$ \\ ${ }^{a}$ University of Georgia, College of Pharmacy, Athens, Georgia \\ ${ }^{\mathrm{b}}$ University at Buffalo, School of Pharmacy and Pharmaceutical Sciences, Buffalo, New York \\ ${ }^{\mathrm{c}}$ Butler University, College of Pharmacy and Health Sciences, Indianapolis, Indiana \\ ${ }^{\mathrm{d}}$ Midwestern University, College of Pharmacy - Downers Grove, Downers Grove, Illinois \\ ${ }^{\mathrm{e}}$ Wingate University, School of Pharmacy, Wingate, North Carolina \\ ${ }^{\mathrm{f}}$ University of Missouri - Kansas City, School of Pharmacy, Kansas City, Missouri \\ g Albany College of Pharmacy and Health Sciences, Albany, New York \\ ${ }^{\text {h }}$ University of California San Diego Health, San Diego, California
} Griffin, PharmD, ${ }^{\mathrm{d}}$ Nicole M. Lodise, PharmD, ${ }^{\mathrm{g}}$ Jaini Patel, PharmD, ${ }^{\mathrm{d}}$ Sally Rafie, PharmD, ${ }^{\mathrm{h}}$ Kathleen Vest,

Corresponding Author: Rebecca H. Stone, University of Georgia, College of Pharmacy, 250 W Green St., Athens, GA 30602. Tel: 404-664-7176. Email: rhstone@uga.edu

Submitted March 29, 2021; accepted August 2, 2021; ePublished August 2021

Starting in 2014, the pharmacist's role in the United States (US) has expanded to include prescribing hormonal contraception (HC), and is currently addressed with policy in 14 states and Washington D.C.. Training and education requirements for this expanded scope of practice vary between states and are changing rapidly. The objective of this review is to examine how student pharmacists are taught to provide contraceptive care, specifically for prescribing ongoing HC and emergency contraception, and to identify potential gaps in current US pharmacy curricula. Despite steady adoption into community pharmacy practice, currently there is sparse literature assessing educational methods used to teach contraceptive care. This review offers recommendations to promote consistent and comprehensive student pharmacist education in providing contraceptive care across institutions, regardless of state policy status.

Keywords: pharmacist prescribed, hormonal contraception, emergency contraception, student pharmacist, teaching method, assessment method

\section{INTRODUCTION}

In the United States (US) the pharmacist's role in reproductive care continues to expand as states broaden the scope of practice to include prescribing hormonal contraception (HC). As of 2021, 14 states and Washington D.C. have specifically authorized pharmacists to prescribe hormonal contraceptives either under statewide protocol, standing order, or specific collaborative practice agreement, with several other states allowing this practice under broader policies. ${ }^{1}$ These efforts build on prior state efforts to increase direct access to emergency contraception (EC) in pharmacies, as well as other pharmacy-based public health interventions such as immunizations, tobacco cessation, and naloxone access. ${ }^{2}$

In a 2009 survey assessing women's perception of pharmacist-prescribed $\mathrm{HC}, 67 \%$ of women perceived not requiring a visit to the physician and incurring a copay as a benefit, while $85 \%$ agreed that convenience of multiple locations and extended hours of community pharmacies make it a preferred avenue for $\mathrm{HC}^{3}$ Additionally, surveys of US pharmacists and student pharmacists beginning in 2009 identify strong interest in prescribing HC in community pharmacies. ${ }^{4,5,6}$ With both patients and pharmacists in strong support of pharmacist-prescribed $\mathrm{HC}$, it is expected to become standard of practice in community pharmacy settings in most, if not all, states in the future. Given this, providing competent contraception care should be an essential core competency within pharmacy education and training.

A study by Rim et al. in 2020 assessed delivery of contraceptive topics and noted that the majority ( 70\%) of doctor of pharmacy (PharmD) programs indicated their curriculum adequately covered contraceptive topics; however, programs also expressed interest in development of a standardized contraceptive curriculum. ${ }^{7}$ Other studies between 2018 and 2020 have highlighted institution specific training opportunities that demonstrate student competence of contraceptive 
knowledge in simulated scenarios and are discussed below, ${ }^{8,9}$ but studies assessing a broader cohort of learners from multiple institutions have not been conducted. Although pharmacists and student pharmacists indicate in surveys that they are comfortable prescribing HC, a desire for additional training in specific areas has also been consistently identified. For example, in 2014, Rafie et al found student pharmacists in California expressed a desire for additional training in product selection, switching between products, and prescribing for minors. ${ }^{10}$ Another study by Stone et al. in 2020 assessing pharmacist readiness demonstrated similar results, with a majority of participants indicating they desired additional training regarding product selection and switching between products. ${ }^{11}$ These surveys highlight potential curricular gaps and opportunities to expand pharmacy education with a consistent approach across colleges and schools of pharmacy.

This review evaluates the available literature describing how pharmacists are taught to provide contraceptive care, specifically for prescribing ongoing EC and HC, and identify potential gaps in the current US PharmD curricula based on this information. In addition, this review offers recommendations to promote consistent and comprehensive student pharmacist education in providing contraceptive care across institutions. Accordingly, a scoping literature search was conducted in Pubmed to identify articles assessing the delivery of contraception education for student pharmacists, physicians, and nurses between 2000 to present.

\section{FINDINGS}

\section{Current Contraceptive Curricula and Student Confidence}

A 2020 survey by Rim et al. assessing the contraception curricula taught in all US pharmacy schools, 100\% of schools that responded indicated that they provided education on $\mathrm{EC}$ and $\mathrm{HC}{ }^{7}$ The majority of respondents also offered non-hormonal over-the-counter (OTC) contraception content (96\%) and long acting reversible contraception (LARC) content $(91 \%)$. On average, colleges or schools of pharmacy spent $6.8 \pm 3.7$ hours on all contraception topics (range: 1.4 22 hours) with EC and HC contributing $0.9 \pm 0.5$ hours (range: 6 minutes - 2 hours) and $2.9 \pm 2$ hours (range: 45 minutes 12 hours) in the curricula, respectively. Patient cases were the most frequent didactic supplement utilized. Despite inclusion of routine and emergency contraceptive content in the vast majority of PharmD programs, research suggests that current curricula may not prepare all graduates to confidently prescribe HC. ${ }^{10-12}$

In 2007 Evans et al. was one of the first studies to evaluate learning outcomes associated with contraception content in pharmacy education ( $n=752$ from 18 colleges of pharmacy). This study evaluated general student knowledge of $\mathrm{EC}$ at different timepoints during the PharmD curriculum, but did not assess a specific learning experience. The results found that student EC knowledge increased with pharmacy school academic year, after taking a course covering contraception, or when EC was a specific topic in the course. ${ }^{13}$ Out of nine questions, the mean knowledge score was 5.9 $(\mathrm{n}=65.5 \%)$. Scores improved with each academic year: first/second year students scored an average of 5.4, third year students scored an average of 5.8 and fourth year students scored an average of 6.4. Evans et al. did not address instructional time or quality of instruction as a component of student knowledge.

In 2018 Hohmann et al. conducted a cross-sectional survey of first-year student pharmacists $(n=110)$ regarding contraceptive knowledge, perceptions, and counseling intentions among students who had not yet received training on contraceptive knowledge in the pharmacy curriculum. ${ }^{12}$ The results of the survey suggested that student pharmacists early in training preferred to learn by watching examples of pharmacists counseling patients on contraception (56\%) and followed by learning through "role-playing" (30\%). In 2014 Rafie et al. conducted a survey of student pharmacists $(\mathrm{n}=502)$ in California prior to the policy change. The survey was administered to student pharmacists who had completed pharmacotherapeutic coursework but not yet started advance pharmacy practice experiences (APPEs). Despite 94\% of students reporting they felt confident to counsel patients on the proper use of $\mathrm{HC}$, only $65 \%$ reported feeling adequately educated to prescribe $\mathrm{HC}$ in their pharmacy practice. ${ }^{10}$ In 2020 Stone et al reported 58\% of pharmacists felt they received adequate training to prescribe $\mathrm{HC}$ after their pharmacy school curricula and post-graduate training. However, a smaller subset felt inadequately trained and indicated that $\mathrm{HC}$ was inadequately covered in their pharmacy school curriculum. Like student pharmacists, a majority of pharmacists indicated that they desired additional training, specifically on switching between contraceptive products and selection of contraceptive products for a specific patient. ${ }^{11}$

Pharmacists are not the only health care providers to report deficits in contraceptive training. Surveys of primary care providers reveal that medical students also report a lack of knowledge and comfort with contraception counseling, ${ }^{14}$ and surveys of graduating medical residents demonstrated an inconsistent contraception knowledge base. ${ }^{15}$ Emergency contraception knowledge follows a similar pattern. In a 2016 survey of prescribers who provided care for women of reproductive age, more effective methods of EC (oral ulipristal acetate and copper intrauterine device) were largely unknown and rarely utilized by general practitioners. ${ }^{16}$ Establishing a standardized curricula for contraceptive training to address these knowledge gaps has been suggested for medical students, but to the authors knowledge has not been developed to date. ${ }^{14}$ 
Similar to the reported US PharmD curricula, the amount of time dedicated to contraceptive training also varies in medical education. A 2003 survey of US and Canadian medical schools demonstrated that most teach 3 to 10 hours of sexual education content and the majority was lecture-based. ${ }^{17}$ Some experts have suggested that training and assessment are lagging behind the clinical and scientific advancements in the field of sexual health. ${ }^{18}$ A report issued in 2010 by the International Consultation of Sexual Medicine, an international, multidisciplinary expert panel, indicates that sexual health training of physicians is inadequate and standardized assessments of clinical skills are needed. ${ }^{18}$

\section{Teaching and Assessment Methods for Contraception Prescribing}

There are limited studies which assess the quality of instructional methods and associated clinical competencies for EC and HC curricula in pharmacy education. The available literature is described below, and a summary of the study objectives, teaching modalities, type of course, study design, and assessment methods can be found in Table 1.

\section{Emergency Contraception}

In 2013 Young et al.(n=189) found that approximately $25 \%$ of students stated they were confident in counseling patients regarding EC prior to an active-learning exercise compared to 59\% after $(p<.01) .{ }^{20}$ Students were asked to rate the degree to which certain barriers hindered their ability to counsel on EC utilizing a scale of 0 to 100 , with 0 representing "not a barrier" and 100 representing "it's a definite barrier." The most common barrier to counseling identified by students on the pre- and post-workshop survey was lack of knowledge (presurvey mean score 41.0 and postsurvey mean score 19.8, $p<001$ ). Student pharmacists' knowledge of counseling patients regarding EC increased from $86 \%$ to $93 \%$ after the exercise $(p<.01)$. The study concluded that active learning exercises may be an effective way to increase student pharmacists' knowledge and confidence regarding EC.

\section{Ongoing Contraception}

A few studies have evaluated teaching methods for contraception training in student pharmacists. Two studies in elective courses have been conducted. A 2018 study by Lynch et al. assessed student pharmacists enrolled in a women's health elective $(n=11) .{ }^{21}$ The students underwent training on state $\mathrm{HC}$ prescribing legislation and completed three simulated standardized-patient scenarios with a prescribing algorithm to make clinical decisions and interact with the simulated patients. Overall, students received an average score of 27.6 out of 30 points, and all completed the activity within the allocated 20 minutes. Students reported overall satisfaction with the activity, generally agreed that the activity was realistic, and reported feeling prepared to prescribe contraceptives. A second study evaluating a women's health elective was conducted by El-Ibiary et al. in 2018 and included contraception discussion activities and active learning in the form of journal article reviews and use of drug information resources. ${ }^{22}$ Of the students enrolled ( $\mathrm{n}=33$ ), $80 \%$ preferred OTC workshops with products as a mode of learning more than any other methods. HC workshops were the second most preferred (61\%) mode of learning. Students' confidence in counseling on women's reproductive health significantly increased after the course (mean scores 2.62 pre-course vs. 3.75 post-course; $p<.01$ ). Students' perceptions of their overall knowledge in women's health also increased (mean scores 2.63 pre-course vs. 3.92 post-course; $p<.01$ ). Prior to the course, students were least confident in counseling on the progestin-only implant and most confident in counseling on EC. After the course, students' confidence increased for both products, respectively (mean score of 1.82 to $3.81 ; 3.70$ to $4.29 ; p<.01$ for both items).

Two larger studies evaluating teaching methods for contraception training in student pharmacists have been conducted. The first was by Harris et al. in 2020, and evaluated student knowledge and confidence with prescribing HC following a simulated patient case activity. ${ }^{8}$ Student performance on an individual readiness assurance test was consistent, with a mean of $84 \%$ for two academic years sampled (2017-2018; $n=131$ and 2018-2019; $n=112)$. Students scored an average of $84 \%$ (2017-2018) and 92\% (2018-2019) on the documented assessment and plan $(p<.001)$ and 96\% (2017$2018)$ and $91 \%(2018-2019)$ on the post-activity quiz $(p=.001)$. Tasks associated with highest confidence included navigating and interpreting the patient's prescription drug formulary, providing the patient with written documentation of the product, and notifying the patient's primary care provider of any product prescribed to the patient. Students reported less confidence with the ability to select an appropriate HC product based on patient-specific factors and providing education on managing missed doses. The second study by Lynch et al. again assessed the impact of a stimulated activity on second year student pharmacist readiness and confidence in prescribing contraception $(n=216) .{ }^{23}$ The mean activity score was $86 \%$ (median 90\%), with a significant change in the students' confidence in their ability to complete the process after the activity $(p<.01)$. The majority of students at baseline $(52 \%)$ and follow up $(53 \%)$ reported needing more practice during Advanced Pharmacy Practice Experiences (APPE) to feel prepared. There was a significant change in the 
number of students who agreed that their curriculum prepared them pre-activity versus post-activity ( $15 \%$ to $29 \%$; $p<$ $.01)$.

Evaluation of teaching activities and learning assessments for contraception training in other health care professions is also limited, but available data indicate that active learning activities may be effective in improving knowledge and comfort with contraceptive care. For example, one 2019 study provided internal medicine residents with a pocket card and one-hour didactic lecture and then assessed their confidence in counseling on and prescribing contraception pre/post the activity. ${ }^{24}$ Forty-two students (56\%) completed the pre-survey and 40 students (53\%) completed the postsurvey. Although there was a significant improvement in resident attitudes towards applicability of contraception training $(p=.03)$, there was no difference in confidence in prescribing or counseling patients regarding oral contraceptives $(p=.05, p=0.45)$. Another 2019 study evaluated internal medicine residents $(\mathrm{n}=38)$ who completed a contraception case-based assessment utilizing interactive online resources (CDC MEC and Reproductive Health Access Project websites) and smartphone applications (CDC Contraception and Contraception Point-of-Care applications). ${ }^{25}$ The mean knowledge score improved significantly after this learning activity, nearly doubling from $49 \%$ correct answers on the pre-survey to $85 \%$ correct on the post-survey. Students were asked to rate their confidence and comfort level with counseling and assessing eligibility of contraception utilizing the following scale: $(1=$ very comfortable/confident, $5=$ not at all comfortable/confident). Post-surveys demonstrated significant improvements in all categories (comfort with counseling, confidence in evaluating medical eligibility, and confidence in initiating contraception. Improvements in confidence were maintained on follow-up surveys four to six months later (evaluating medical eligibility presurvey mean score 3.2 and delayed postsurvey mean score $2.7, p=.004$ ) and initiating the pill, patch, ring, or injection (presurvey mean score 3.6 and delayed postsurvey mean score 3.0, $p=.002$ ).

\section{Potential Barriers to Contraception Curricula Delivery}

One major limitation in evaluating teaching activities for student pharmacist contraception training is the understanding that there is a discordance between methodologies actively being utilized in the classroom and what is represented in published work. Robust literature related to active learning techniques, applied skills lab time within the curriculum, and simulation scenarios is lacking. Educators are likely utilizing these techniques; however, they are not publishing their creative work. This can be due to a variety of reasons including lack of time or concerns of lack of rigor or high enough innovation for peer consumption.

Faculty may face barriers that impact successful implementation and expansion of advanced contraception education into pharmacy curricula. ${ }^{7,26,27}$ Some of the overarching concerns that commonly impact curricular development include faculty resources or resources for activity development and curricular design. School curricula are often already strained for space and time, and inserting a new activity can be challenging. Additionally, having the faculty resources for development of new activities may be difficult depending on the institution and faculty expertise. Depending upon institutional core beliefs, there may also be limitations placed upon how in depth contraception content can be delivered, discussed, and applied. When extrapolating data from other health professions similar barriers have been documented. ${ }^{24-28}$ Barriers in delivering sexuality content, including contraception, within nursing contraceptive curricula have been documented and include: time constraints in the curriculum, less priority compared to other topics, comfort and capability to teach the topics, and importance not seen by faculty. ${ }^{26,27}$ Aaberg found $40 \%$ of responding nursing programs identified time constraints as the primary barrier in 2016 , which aligns with a study nearly 50 years prior in which $45 \%$ identified time as a barrier. ${ }^{26,28}$ Colleges and schools of pharmacy also identified time as a barrier to contraception education curricula incorporation in the 2020 study by Rim et al, although $68 \%$ felt the curriculum was adequate. Only $29 \%$ of colleges or schools of pharmacy agreed incorporating six to eight hours of $\mathrm{HC}$ education into the required didactic curriculum would be easy. Interestingly, $61 \%$ agreed providing six to eight hours of contraception education would be easy in the elective curriculum. ${ }^{7}$ Although expanding women's health education within any part of a curriculum should be lauded, elective education expansion alone would not contribute to the practice-readiness of all students in a pharmacy program.

It is important to note that the optimal duration of instructional time needed to deliver contraceptive content is unknown, and instructional time may not directly correlate with an increase in practice-readiness. Typical assumptions often include the idea that additional instruction time results in increases in performance outcomes. A 2017 study by Cattaneo et al. in secondary education illustrated that an additional hour of instruction only delivered 30-40\% of the expected learning impact that is typically associated with the first hour of instruction. ${ }^{29}$ In 2015 Lavy and Rivkin and Schiman, like Cattaneo et al., found increased instructional time modestly increased performance on examinations in secondary education. ${ }^{29-31}$ Both studies noted the differences were related to instructional time productivity and quality with better productivity and higher quality having larger gains..$^{30,31}$ 
Depending on state regulations and current contraception prescribing practices, pharmacy faculty, preceptors, and students may have different expectations of the importance of advanced student learning activities within the curriculum in regards to contraception. Theoretically, students and educators located in states that provide pharmacists the opportunity to prescribe may place a greater emphasis on active learning and simulated activities and devote more time to the topic. Additionally, lack of support for these types of activities from other health care providers may exist as well, which could impact interprofessional education experiences. The most commonly used learning technique for contraception in the 2020 study by Rim et al was the use of case studies, but the lowest utilized method was patient interviews. ${ }^{7}$ The use of active learning techniques allows students to apply the information they have learned and grow in their comfort level with prescribing contraception. Rim et al. suggested that patient interview utilization could be increased to aid with student confidence. As practicing pharmacists often cite training as a barrier to providing this service, robust activities using a variety of teaching techniques can help best equip students for practice. ${ }^{32}$

Student attitudes towards contraception prescribing also play a role in the intent to perform this action. In 2018, Hohmann et al. discovered that among first-year student pharmacists who had not yet learned about contraception, those with more positive attitude scores regarding contraception counseling had a higher intent to counsel. It is possible that perceived importance of contraceptive education may vary between students, schools of pharmacy (ie. religious versus secular institutions), and states (ie, pharmacist prescribing versus non-prescribing). These factors may ultimately influence uptake and availability of pharmacist prescribed contraception.

The majority of states which allow pharmacist-prescribed contraception mandate completion of a training course, which varies among states. Some states waive this requirement for those who graduated from a school in the state after the bill went into effect. See Table 2. It is unclear if receiving this prescriptive authority as part of the PharmD degree instead of post-graduate training increases the number of pharmacists who choose to provide this service.

\section{DISCUSSION \\ Recommendations}

Data indicate that all student pharmacists currently receive contraceptive care education; however, studies have identified that a subset of students report they do not feel adequately prepared to prescribe contraception or desire additional training in specific areas. ${ }^{7}$ There is limited literature assessing the specific teaching modalities that are currently employed in colleges and schools of pharmacy to deliver this content, particularly in the context of the pharmacy professions expanding scope of practice. The majority of available literature assesses student self-perception or confidence, which likely does not fully assess if a teaching methodology is effective without accompanying outcomes data. Additionally, the authors did not identify any data specifically assessing contraceptive care education within introductory pharmacy practice experiences (IPPE) or advanced pharmacy practice experiences (APPE) training.

The Accreditation Council for Pharmacy Education (ACPE) set standards for PharmD programs in the US and address three key standards within the required Educational Outcomes: 1) Foundational Knowledge, 2) Essentials for Practice and Care, and 3) Approach to Practice and Care ${ }^{33}$ The pharmacist role in providing contraceptive care has changed rapidly since 2013. In this quickly changing landscape, PharmD curricula will require review and potential revision in order to prepare graduates to confidently prescribe contraception, a skill that is becoming essential in community pharmacy practice.

The American College of Clinical Pharmacy's (ACCP) Pharmacotherapy Didactic Curriculum Toolkits have strongly recommended contraception education as a tier 1 core curriculum component within the Women's Health section since the first of three iterations in 2009..$^{34-36}$ The tier 1 recommendation is defined as topics in which education and training should prepare learners to provide patient-centered, collaborative care by demonstrating competence during or at the end of the program. The American Association of Colleges of Pharmacy (AACP) Learning and Performance Outcomes also address contraception education, stating that students should be able to "develop appropriate pharmaceutical care plans for pregnancy prevention." ${ }^{37}$ In 2020 Rim et al. found that all responding PharmD programs reported inclusion of $\mathrm{HC}$ and $\mathrm{EC}$ in their curricula, as recommended by these organizations. ${ }^{7,36,37}$ However, only $68 \%$ of responding institutions believe the contraception education regarding LARCs is adequate. ${ }^{7}$ As noted earlier, a majority of surveyed student pharmacists and pharmacists indicated they would like additional product specific education in preparation for prescribing contraception. ${ }^{10,11}$ This data suggest that the essential content needed to provide patientcentered contraceptive care may not be uniformly met by all PharmD programs. ${ }^{34}$

Although colleges and schools of pharmacy include contraceptive content in the didactic portion of their curricula, few utilize more complex teaching modalities such as simulated patient cases. Data are limited regarding learner outcomes associated with instructional time, content delivery, and assessment of practice-readiness in pharmacy contraception education. To the authors' knowledge, research has not been conducted to assess if there is a relationship between the type 
of teaching activity (ie, SOAP note, case studies, role play, simulated patient) and the outcome of interest - graduating a practice ready pharmacist, prepared to prescribe contraceptive products. Other health care professions face similar challenges in delivering sexual education and reproductive health content. ${ }^{14,15,19}$ Medical schools often use lecture-based methods to teach these topics, with data indicating this may not effectively prepare students to confidently practice in this area. ${ }^{16,24}$ However, evaluations of more active learning styles indicate they increase student interest, confidence, and have a sustained effect. ${ }^{38}$ Both pharmacy and medical student educators have identified that standardization of learning activities may improve training in their field, through either standardization of curricula or assessments of clinical skills. ${ }^{7,18}$

As the role of the pharmacist continues to expand in contraceptive care, the authors put forth the following recommendations for educators to consider when teaching regarding pharmacist prescribing of contraception and emergency contraception:

1) Doctor of Pharmacy programs should proactively address curriculum and experiential training gaps that may develop with the rapidly expanding pharmacist scope of practice, such as hormonal contraception prescribing.

2) Graduating pharmacists should have a working knowledge of the pharmacology of all contraceptive products available, the ability to efficiently access and apply more detailed product specific information, and the clinical skills to provide direct contraception care.

3) Pharmacy school curricula should incorporate key guidelines in providing contraceptive care, such as the United States Medical Eligibility Criteria for Contraceptive Use ${ }^{39}$ and the United States Selected Practice Recommendations for Contraceptive Use. ${ }^{40}$

4) Graduating pharmacists should possess communication skills which allow them to gather pertinent and often sensitive patient information, and apply their knowledge to provide contraceptive education and care for a specific patient.

5) Doctor of pharmacy programs may consider working directly with state regulatory agencies to ensure pharmacists are prepared and authorized upon graduation to prescribe contraceptives, which may aid implementation of this service for state residents to access.

6) Doctor of pharmacy programs may consider including discussion regarding potential barriers and facilitators to implementation of pharmacist prescribing of contraception within the curricula (eg, payment structure, overcoming resistance from other providers)

7) Pharmacy educators are encouraged to evaluate and publish data regarding novel training techniques used to teach contraceptive care.

\section{CONCLUSION}

An increasing number of states now permit pharmacist prescribing of select medications, including HC and EC. The training that student pharmacists receive must also adapt in order to produce "practice ready" graduates in this changing landscape. Additional research is needed to identify which types of teaching methods are most effective in preparing graduating pharmacists to confidently prescribe contraceptives, and then utilized to translate best teaching practices into pharmacy education.

\section{ACKNOWLEDGMENTS}

\section{Disclosures}

Kylie Barnes - Consultant, Wolter's Kluwer

Sally Rafie - Clinical Advisory Board, Afaxys Inc.; Speaker, TherapeuticsMD

\section{REFERENCES}

1. Birth Control Pharmacist. Policies - State policies and protocols for pharmacist prescribing of contraception. https://birthcontrolpharmacist.com/policies/.

2. National Alliance of State Pharmacy Associations (NASPA). Scope of Practice. https://naspa.us/restopic/scope/. Accessed March 25, 2021.

3. Landau SC, Tapias MP, McGhee BT. Birth control within reach: a national survey on women's attitudes toward and interest in pharmacy access to hormonal contraception. Contraception. 2006;74(6):463-470.

4. Landau S, Besinque K, Chung F, et al. Pharmacist interest in and attitudes toward direct pharmacy access to hormonal contraception in the United States. J Am Pharm Assoc (2003). 2009;49(1):43-50.

5. Rafie S, Cieri-Hutcherson NE, Frame TR, et al. Pharmacists' Perspectives on Prescribing and Expanding Access to Hormonal Contraception in Pharmacies in the United States. J Pharm Pract. 2019:897190019867601. 
6. Rafie S, El-Ibiary SY. Student pharmacist perspectives on providing pharmacy-access hormonal contraception services. J Am Pharm Assoc (2003). 2011;51(6):762-765.

7. Rim C, El-Ibiary SY, Rafie S, Borgelt LM. Assessment of contraceptive curricula in US pharmacy programs. Curr Pharm Teach Learn. 2020;12(4):395-399.

8. Harris E, Melody K, Charneski L. Student knowledge and confidence with prescribing hormonal contraception. Curr Pharm Teach Learn. 2020;12(6):751-762.

9. Lynch SE, Griffin BL, Vest KM. Assessment of a simulated contraceptive prescribing activity for pharmacy students. Currents in Pharmacy Teaching and Learning. 2018;10(2):178-184.

10. Rafie S, El-Ibiary, SY. California Pharmacy Student Perceptions of Confidence and Curricular Education to Provide Direct Pharmacy Access to Hormonal Contraception. Pharmacy Education. 2014;14(1):31-36.

11. Stone RH, Rafie S, Griffin B, Shealy K, Stein AB. Pharmacist self-perception of readiness to prescribe hormonal contraception and additional training needs. Curr Pharm Teach Learn. 2020;12(1):27-34.

12. Hohmann N, Kavookjian J. Using the Theory of Planned Behavior to determine pharmacy students' intention to participate in hormonal contraception counseling services. Curr Pharm Teach Learn. 2018;10(11):1488-1495.

13. Evans E, Patel M, Stranton D. Student pharmacist knowledge and attitudes regarding oral emergency contraception. J Am Pharm Assoc (2003). 2007;47(6):711-716.

14. Akers AY, Gold MA, Borrero S, Santucci A, Schwarz EB. Providers' perspectives on challenges to contraceptive counseling in primary care settings. J Womens Health (Larchmt). 2010;19(6):1163-1170.

15. Schreiber CA, Harwood BJ, Switzer GE, Creinin MD, Reeves MF, Ness RB. Training and attitudes about contraceptive management across primary care specialties: a survey of graduating residents. Contraception. 2006;73(6):618-622.

16. Batur P, Cleland K, McNamara M, Wu J, Pickle S, Group ECS. Emergency contraception: A multispecialty survey of clinician knowledge and practices. Contraception. 2016;93(2):145-152.

17. Solursh DS, Ernst JL, Lewis RW, et al. The human sexuality education of physicians in North American medical schools. Int J Impot Res. 2003;15 Suppl 5:S41-45.

18. Eardley I, Reisman Y, Goldstein S, Kramer A, Dean J, Coleman E. Existing and Future Educational Needs in Graduate and Postgraduate Education. J Sex Med. 2017;14(4):475-485.

19. Parish SJ, Rubio-Aurioles E. Education in sexual medicine: proceedings from the international consultation in sexual medicine, 2009. J Sex Med. 2010;7(10):3305-3314.

20. Young S, Griffin B, Vest K. Active-learning instruction on emergency contraception counseling. Am J Pharm Educ. 2013;77(5):104.

21. Lynch SE, Griffin BL, Vest KM. Assessment of a simulated contraceptive prescribing activity for pharmacy students. Curr Pharm Teach Learn. 2018;10(2):178-184.

22. El-Ibiary SY, Azizoddin A, Petrossian L, Gurney MK. Implementation and evaluation of a women's reproductive health pharmacy elective. Curr Pharm Teach Learn. 2018;10(8):1087-1096.

23. Lynch SE, Griffin B, Stewart-Lynch A, Vest KM. Effect of a Simulated Activity on Student Ability, Preparedness, and Confidence in Applying the Pharmacists' Patient Care Process to Contraceptive Prescribing. Pharmacy (Basel). 2020;8(3).

24. Maciuba JM, Chen JM. A New Contraception Curriculum in the Internal Medicine Residency Primary Care Clinic. Mil Med. 2019;184(1-2):e61-e64.

25. Bachorik A, Nemer MK, Chen GL, et al. Case-Based Curriculum With Integrated Smartphone Applications Improves Internal Medicine Resident Knowledge Of Contraceptive Care. Adv Med Educ Pract. 2019;10:971-977.

26. Aaberg V. The state of sexuality education in baccalaureate nursing programs. Nurse Educ Today. 2016;44:14-19.

27. Astbury-Ward E. A questionnaire survey of the provision of training in human sexuality in schools of nursing in the UK. Sex Relation Ther. 2011;26(3):254-270.

28. Woods NF, Mandetta AF. Sexuality in the baccalaureate nursing curriculum. Nurs Forum. 1976;15(3):294-313.

29. Cattaneo MA, Oggenfuss C, Wolter SC. The more, the better? The impact of instructional time on student performance. Education Economics. 2017;25(5):433-445.

30. Lavy V. Do Differences in Schools' Instruction Time Explain International Achievement Gaps? Evidence from Developed and Developing Countries. The Economic Journal. 2015;125(588):F397-F424.

31. Rivkin SG, Schiman JC. Instruction time, Classroom Quality, and Academic Achievement. The Economic Journal. 2015;125(588):F425-F448.

32. Lin CW, Chang EH, Clinciu DL, et al. Using modified information delivery to enhance the traditional pharmacy OSCE program at TMU - a pilot study. Comput Methods Programs Biomed. 2018;158:147-152. 
33. Accreditation Council for Pharmacy Education (ACPE). Accreditation Standards and Key Elements for the Professional Program in Pharmacy Leading to the Doctor of Pharmacy Degree, 2016. 2015; https://www.acpeaccredit.org/pdf/Standards2016FINAL.pdf Accessed March 25, 2021.

34. American College of Clinical Pharmacy (ACCP). Pharmacotherapy Didactic Curriculum Toolkit 2009. http://www.accp.com/docs/positions/misc/pharmacotherapytoolkit.pdf. Accessed March 25, 2021.

35. Schwinghammer TL, Crannage AJ, Boyce EG, et al. The 2016 ACCP Pharmacotherapy Didactic Curriculum Toolkit. Pharmacotherapy. 2016;36(11):e189-e194.

36. Flannery AH, Soric MM, Benavides S, et al. 2019 Update to the American College of Clinical Pharmacy Pharmacotherapy Didactic Curriculum Toolkit. Journal of the American College of Clinical Pharmacy. 2020;3(2):455-464.

37. American Association of Colleges of Pharmacy (AACP) Learning and Performance Outcomes: Women's Health Learning Outcomes. https://www.aacp.org/resource/learning-and-performance-outcomes Accessed July 20, 2021.

38. Rosen R, Kountz D, Post-Zwicker T, Leiblum S, Wiegel M. Sexual communication skills in residency training: the Robert Wood Johnson model. J Sex Med. 2006;3(1):37-46.

39. Curtis KM, Tepper NK, Jatlaoui TC, et al. U.S. Medical Eligibility Criteria for Contraceptive Use, 2016. MMWR Recomm Rep. 2016;65(3):1-103.

40. Curtis KM, Jatlaoui TC, Tepper NK, et al. U.S. Selected Practice Recommendations for Contraceptive Use, 2016. MMWR Recomm Rep. 2016;65(4):1-66. 
Table 1. Literature Assessing Contraception Teaching Modalities and Outcomes

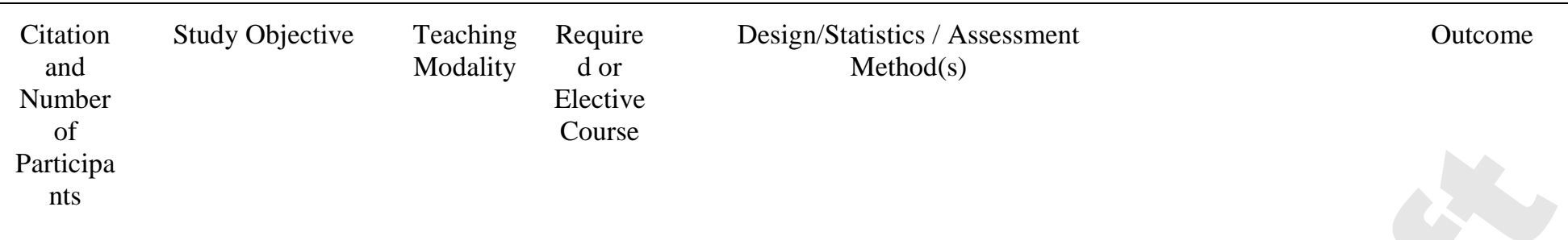

Student Pharmacist Outcomes

\begin{tabular}{|c|c|c|c|c|c|}
\hline $\begin{array}{l}\text { Young } \\
2012 \\
n=189\end{array}$ & $\begin{array}{l}\text { To increase } \\
\text { pharmacy students' } \\
\text { knowledge of and } \\
\text { confidence in } \\
\text { counseling patients } \\
\text { regarding EC and to } \\
\text { identify any barriers } \\
\text { to counseling } \\
\text { patients about EC }\end{array}$ & $\begin{array}{l}\text { Worksho } \\
\mathrm{p}\end{array}$ & $\begin{array}{l}\text { Require } \\
\text { d }\end{array}$ & $\begin{array}{l}\text { - Cross-sectional pre and post } \\
\text { workshop survey } \\
\text { - Students observed a 5-minute skit of } \\
\text { a counseling session on EC and then } \\
\text { were asked to pair up with a } \\
\text { classmate and practice counseling } \\
\text { each other regarding the use of EC } \\
\text { following a checklist of key points }\end{array}$ & $\begin{array}{l}\text { - } 94.5 \% \text { students responded } \\
\text { - Students' knowledge scores increased from } 86 \% \text { to } \\
93 \%(\mathrm{p}<.01) \\
\text { - } \text { Approximately } 25 \% \text { of the students stated they } \\
\text { were confident in counseling patients on EC prior } \\
\text { to active-learning exercise compared to } 58.5 \% \\
\text { after ( }<.01) \\
\text { The most common barrier to counseling that } \\
\text { students identified on the pre- and post-workshop } \\
\text { survey was lack of knowledge. }\end{array}$ \\
\hline $\begin{array}{l}\text { Lynch } \\
2018 \\
n=11\end{array}$ & $\begin{array}{l}\text { Determine the } \\
\text { utility of a } \\
\text { contraceptive } \\
\text { prescribing } \\
\text { simulation activity } \\
\text { for pharmacy } \\
\text { students }\end{array}$ & $\begin{array}{l}\text { Simulatio } \\
\mathrm{n}\end{array}$ & Elective & $\begin{array}{l}\text { - Cross-sectional post activity survey } \\
\text { Faculty graded clinical decision- } \\
\text { making based on the student's } \\
\text { electronically submitted A/P. } \\
\text { - The SP graded interpersonal skills } \\
\text { using the CSC's standardized } \\
\text { communication skills rubric. }\end{array}$ & $\begin{array}{l}\text { - } 100 \% \text { students completed the activity in the } \\
\text { allocated } 20 \text { min } \\
\text { Overall students received an average score of } 27.6 \\
\text { out of } 30 \text { points } \\
\text { Students reported overall satisfaction with the } \\
\text { activity, with general agreement that the activity } \\
\text { was realistic and made them feel like they were } \\
\text { prepared to prescribe contraceptives }\end{array}$ \\
\hline
\end{tabular}



and evaluation of an elective course in women's

reproductive health

Didactic

and

Simulatio $\mathrm{n}$

Assess student knowledge and confidence with prescribing $\mathrm{HC}$ following a simulated patient case activity in 2017 to 2018 and 2018 to 2019

academic years
- Paired sample t-tests to compare pre and post course survey

- $\quad$ Self-perceived confidence was measured on a Likert scale

- Quizzes given at the end of each lecture, participation in 3 workshops, 2 take-home case assignments, 1 group project and peer evaluation, and an open notes final exam
Simulatio Require
- $80 \%(n=24)$ of students preferred OTC workshops with products more than any other methods

- Students' confidence in counseling on women's reproductive health significantly increased after the course (mean scores 2.62 pre-course and 3.75 postcourse, $\mathrm{p}<.01)$.

- Students' perceptions of their overall knowledge in women's health (mean scores 2.63 pre-course and 3.92 post-course, $\mathrm{p}<.01$ ).

- Prior to the course, students were least confident in counseling on the progestin-only implant (mean score 1.82) and most confident in counseling on EC (mean score 3.70).

- After the course, students' confidence $\uparrow$ for both products (mean scores 3.81 and 4.29 respectively with $p<.01$ for both items).

- Student performance on the IRAT was consistent from 2017 to 2018 to 2018 to 2019 with a mean score of $83.9 \% \pm 16.3$ and $83.9 \% \pm 17.8$ respectively, $\mathrm{p}=0.979$. data from 2nd professional year students during the 2017 - 2018 \& 2018 - 2019 academic years.

- Student performance was assessed using a five-question readiness assurance test (IRAT) at the beginning of class, documented patient $\mathrm{A} / \mathrm{P}$, and five-question postactivity quiz
- Students scored an average of $84.2 \%$ and $91.6 \%$ (p $<.001)$ on the documented A/P and $96.4 \%$ and

$91.2 \%(\mathrm{p}=.001)$ on the post-activity quiz for each year, respectively.

- Tasks associated with highest confidence included navigating and interpreting the patient's prescription drug formulary, providing the patient with written documentation of the product, and notifying the patient's primary care provider of any product prescribed to the patient.

- Students reported less confidence with the ability to select an appropriate $\mathrm{HC}$ product based on patient-specific factors and providing education on managing missed doses. 
Assess the impact

of a simulated

activity on studen

pharmacists'

readiness for, ability

to use, and

confidence in

applying the PPCP

along with the US

MEC to a patient

seeking

contraception.

Medical Students

Maciuba Elucidate the

2019 perceptions of

$\mathrm{n}=40 \quad$ contraception in an

IM primary care

setting in a military

residency and

improve confidence

in contraceptive

counseling and

prescribing
Simulatio Require

$\mathrm{n}$

- Quasi-experimental study

- Chi-square and Mann-Whitney U tests were used to analyze categorical variables and Likertscale data, respectively.

- Students completed a contraceptiveprescribing simulation with SPs.

- Scores were analyzed for safe and appropriate prescribing practices

- Pre- and post-workshop surveys measured confidence and perceived preparedness.

$\begin{array}{ll}\text { Didactic } & \text { Resident } \\ & \text { s at a } \\ \text { military } \\ \text { IM } \\ \text { residenc } \\ \text { y } \\ \text { program }\end{array}$

\section{- Description}

- 1-hour long presentation on contraception that was followed 5 weeks later by the implementation of a pocket card for point of care use with key information from the presentation.

- Residents became certified in the placement and removal of the etonogestrel implant.

- Pre- and post-intervention surveys were administered to assess for changes in attitudes and confidence in prescribing and counseling.
- $91.2 \%(\mathrm{n}=197)$ students completed the pre survey

- $42.6 \%(n=92)$ students completed the post survey

- Mean activity score was $86 \%$ (median $90 \%$ ), with significant change in student confidence of ability to complete the process $(\mathrm{p}<.01)$.

- Majority of students at baseline (52.2\%) and follow up (53.2\%) reported needing more practice during APPEs to feel prepared.

- There was a significant change pre/post in students who agreed that their curriculum prepared them ( $15 \%$ to $28.7 \%$, p <.01).

- Statistically significant changes in confidence for all aspects of the pharmacist patient care process as related to contraception provided by pharmacist (ex. assessing the patient, making a recommendation, monitoring the patient, referring the patient when needed)

- $53 \%$ (40 of 75) of participants responded.

- There was a significant improvement in resident attitudes towards applicability of contraception training $(\mathrm{p}=.03)$ as well as acquiring the skill of etonogestrel implant placement $(\mathrm{p}<.01)$.

- There was a trend towards significance in confidence in prescribing OC $(\mathrm{p}=.05)$.

- There was no change in residents' confidence in counseling on $\mathrm{OC}(\mathrm{p}=0.45)$ 
Bachorik Evaluate whether a Case-base Require

2019 new contraception

workshop d

$\mathrm{n}=38$ pre- curriculum

survey \& improved IM

$\mathrm{n}=20 \quad$ resident knowledge

post- of and comfort with

survey contraceptive care
- Descriptive

- Pre and post activity survey assessing knowledge of and comfort with contraceptive care

- 2-hour session

- Interns were provided with select online resources and two smartphone applications at the beginning of the session, which they then used in case-based small group work
- 38 paired pre- and immediate post-surveys ( $95 \%$ of possible survey participants) and 20 (50\% of possible survey participants) completed the delayed post-survey 4-6 months after the curriculum.

- Pre-session, the mean knowledge score (eg, \% of 10 questions answered correctly) was $<50 \%$ among all participants

- Mean ratings of comfort and confidence around contraceptive care ranged from 3.0 for comfort with counseling about the IUD or implant to 3.6 for confidence in initiating birth control pills, patch, ring or injection $(1=$ very comfortable or confident \& $5=$ very uncomfortable)

- Post-session, the mean knowledge score improved significantly, nearly doubling from $49.2 \%$ correct answers on the pre-survey to $84.5 \%$ correct on the post-survey

- Post-curriculum surveys demonstrated significant improvements in knowledge about comfort with counseling about assessing medical eligibility for and initiating multiple forms of contraception.

- Improvements in knowledge and comfort were maintained on follow-up surveys 4-6 months later

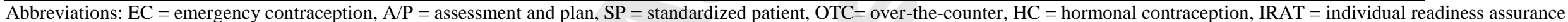

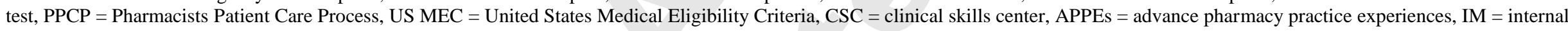
medicine, $\mathrm{OC}=$ oral contraception, $\mathrm{IUD}=$ intrauterine device

Table 2. Training Requirements in States with Regulations Specific to Pharmacist-Prescribed Contraception

\begin{tabular}{|c|c|c|c|c|}
\hline $\begin{array}{l}\text { States with } \\
\text { Pharmacist } \\
\text { hormonal } \\
\text { contraception } \\
\text { prescribing }^{\mathrm{a}}\end{array}$ & $\begin{array}{l}\text { Prescriptive authority } \\
\text { status }\end{array}$ & Training Program Requirements & $\begin{array}{l}\text { Can recent PharmD } \\
\text { graduates prescribe in that } \\
\text { state without additional } \\
\text { training? }\end{array}$ & State Regulations \\
\hline Arkansas & $\begin{array}{l}\text { In development; } \\
\text { Statewide protocol }\end{array}$ & $\begin{array}{l}\text {-Approved by BOP } \\
\text {-Hours not noted }\end{array}$ & Unknown & $\begin{array}{l}\text { https://www.arkleg.state.ar.us/Bills/FTPDoc } \\
\text { ument?path=\%2FBills\%2F2021R\%2FPubli } \\
\text { c\%2FHB1069.pdf }\end{array}$ \\
\hline California & $\begin{array}{l}\text { Implemented; } \\
\text { Statewide protocol }\end{array}$ & $\begin{array}{l}\text {-Approved by BOP } \\
-1 \text { hour }\end{array}$ & Yes; starting in 2014 & $\begin{array}{l}\text { https://www.pharmacy.ca.gov/publications/ } \\
\text { hormonal_contraception_protocol_rphs.pdf }\end{array}$ \\
\hline Colorado & $\begin{array}{l}\text { Implemented; } \\
\text { Statewide protocol }\end{array}$ & $\begin{array}{l}\text {-ACPE-accredited } \\
\text {-Hours not noted }\end{array}$ & No & 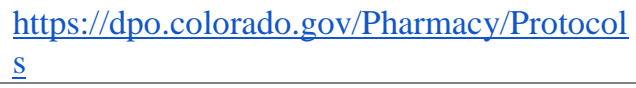 \\
\hline
\end{tabular}




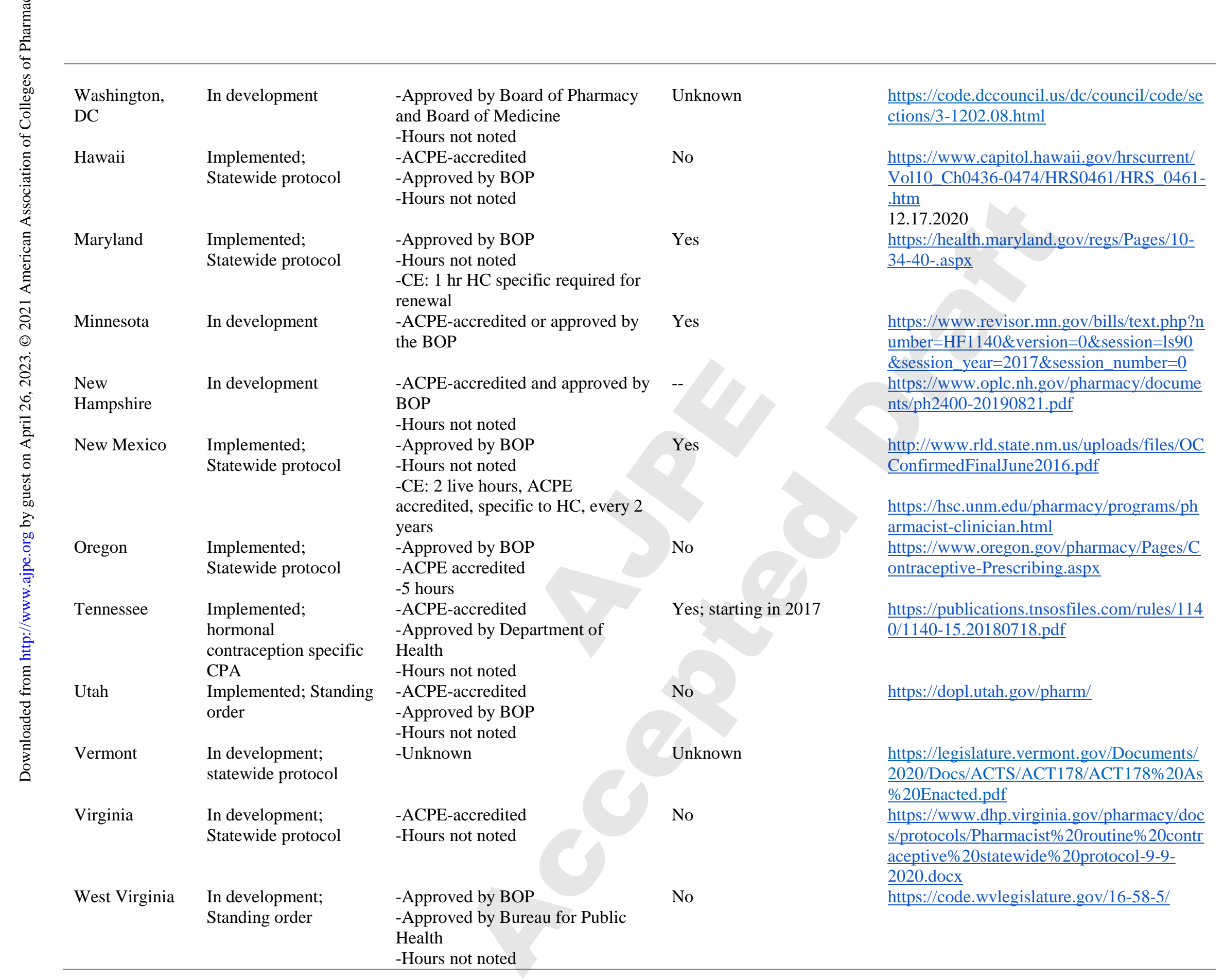


addaho, Michigan, Montana, Washington, allow pharmacists to prescribe contraception under general collaborative practice agreements without specific training requirements.

Abbreviations: $\mathrm{BOP}=$ Board of Pharmacy, $\mathrm{ACPE}=$ Accreditation Council for Pharmacy Education, $\mathrm{HC}=$ hormonal contraception, $\mathrm{CE}=$ continuing education

Birth Control Pharmacist: Policies. https://birthcontrolpharmacist.com/policies/, updated August 18, 2020. Accessed 1/29/2021.

National Alliance of State Pharmacy Associations. Pharmacist prescribing: hormonal contraceptives. Available at: https://naspa.us/resource/contraceptives/ Accessed February 1, 2020. 